\title{
Cosmological impact of winding strings
}

\author{
Jochen Kripfganz $\dagger \ddagger$ and Holger Perlt $\ddagger$ \\ $\uparrow$ Deutsches Elektronen-Synchrotron DESY, Hamburg, Federal Republıc of Germany \\ \& Sektıon Physık, Karl-Marx-Universitat, Leipzig, German Democratıc Republic
}

Recelved 21 July 1987

\begin{abstract}
The thermodynamics and cosmology of torus-compactıfied heterotic strings are studied We emphasise qualitatively new effects due to compactification New topologically stable states appear which correspond to strings winding around the non-simply-connected compact manifold Under reasonable assumptions they avold the blowing up of the compactification scale when the universe becomes matter dominated For a higherdimensional point field theory with scale-invariant ground state this blowing up would be unavordable
\end{abstract}

\section{Introduction}

Superstring theories (Green et al 1987 and references therein) are optımıstically considered as candidates for an ultimate theory of all fundamental interactions of nature, including gravity. These theories are consistent (anomaly-free) chiral quantum theories, incorporate gravity in a natural way, and are presumably finite. As a theory of everything they would also have to allow a derivation of the standard model of strong, electromagnetic and weak interactions, 1 e. determine all the free parameters Including the family number and the observed gauge structure. Furthermore, they would have to explain the vanishing of the cosmological constant despite supersym. metry breakıng (Moore 1987).

At the present level of understanding the best one can say is that this does not appear to be impossible In any case, no attractive alternatives are around, at least none which would attack all these problems at once

One problem with the present formulation of string theories is that it is intrinsically perturbative, with strings moving on a classical background. Many classical solutions (superconformal field theories) exist, and it is unclear how to select the 'right' one. Eventually the theory should self-consistently determine its own background. This could only be expected from a non-perturbative formulation which has not yet been found. Actually solving such a theory in a non-perturbative way would still be another matter

Despite this very incomplete understanding of the structure of the theory it is necessary to work out observable consequences at the level of string perturbation theory. This first of all means looking for classical solutions with acceptable low energy predictions (masses, Yukawa couplings, family number). No completely satisfactory solution has yet been found. The other possible approach to make contact with phenomenology is to study the early history of the universe. Here the most striking prediction of string theories is the existence of a phase transition at a critical temperature 
$T_{c}$ close to the Planck scale. For the heterotic string (Gross et al 1985, 1986) $T_{c}$ is given by

$$
T_{\mathrm{c}}=\left[(2+\sqrt{2}) \pi \alpha^{1 / 2}\right]^{-1}
$$

with $\alpha^{1 / 2}$ a fundamental scale parameter of the order of the Planck length. $T_{\mathrm{c}}$ simply reflects the exponential increase of the level density of a free string gas. The specific heat turns out to be finite as $T$ approaches $T_{\mathrm{c}}$ from below. Therefore $T_{\mathrm{c}}$ does not have the interpretation of a limiting temperature (Hagedorn 1965) but indicates a phase transition. This phase transition actually prevents us from following the evolution of the universe backward in time beyond the Planck time. This, however, would normally be one of the main objectives of a finite quantum theory of gravity This might be another indication that perhaps a completely different formulation of string theories would be needed. The situation might resemble that of the physics of strong interactions before QCD. The existence of a high-temperature phase transition was anticipated from the exponentially rising hadronic mass spectrum but there was no way to understand the nature of the high-temperature phase without the notion of quarks and gluons. Similarly, a radically new approach might be needed starting from string theories, leaving the present formulation as an effective theory, perhaps appropriate for the low-temperature (long-distance) behaviour. The notion of smooth spacetime manifolds might lose its meaning at short distances.

Below the critical temperature the cosmological evolution is modified by higherderivative contributions to the Einstein equation (as well as to the background field equations of other massless modes), and by the contribution of higher string modes to the energy-momentum tensor. Usually these effects are very small, and the cosmo. logical evolution apparently does not differ significantly from that of the point field theory of the corresponding massless modes.

The original approach to string theories in four-dimensional spacetime was to compactify superstrings in their critıcal dimension $d_{c}=10$, i e. looking for classical solutions of topology $M_{4} \times K_{6}$ with $K_{6}$ a six-dimensional compact space. Most studied examples are tori (Narain et al 1987b) or orbifolds (Dixon et al 1985a, b). A general feature of these solutions is scale invariance, 1 e. the compactification scale $R$ is not specified.

One might simply assume $R$ to be small but this leads to a cosmological problem This procedure could only be acceptable if $R$ would remain small once it has been chosen to be so initially. For point field theories this is the case for a radiation. dominated universe but not for matter dominance There $R$ starts to blow up in an unacceptable way (see, e.g., Weiss 1986). It will be the main point of this paper to demonstrate that string theories provide a solution to this problem which is not available in the case of point field theories. It is in fact well known that, for string theories compactified on non-simply connected manifolds, new states appear corresponding to strings winding around the extra dimensions. They form topologically stable sectors and appear as states with an $R$-dependent mass in the equivalent effective point theory For certain values of the compactification scale or other background fields they may become massless (points of enhanced symmetry) To our knowledge these states (called winding states or solitons in the following) have so far not been studied in a cosmological context. We shall show that these states in fact stabilise the compactification radius under reasonable assumptions.

Very recently string theories have been formulated directly in four dimensions. Presumably they cannot be understood through compactification of ten-dimensional 
string theories (Kawai et al 1987, Antoniadis et al 1986, Narain et al 1987a, Lerche et al 1986). For these theories the question of a higher-dimensional Kaluza-Klein type cosmology does not arise, and our solution of providing a stable value for the compactification radius (i.e. one of the background fields) does not apply. Of course, the existence of these theories makes the problem of selecting one vacuum out of many possible ones only more severe.

The outline of this paper is as follows. In $\S 2$ we study the thermodynamics of a free gas of heterotic strings compactıfied on a particular torus. We concentrate on the aspect of compactification because the thermodynamics of uncompactified strings has been studied before (Tye 1985, Bowick and Wijewardhana 1985, Alvarez 1986, Alvarez and Osorio 1986, Matsuo 1986, Gleiser and Taylor 1985). Section 3 represents the main part of this paper. We present numerical estımates on the stability range of a four-dimensional or ten-dimensional cosmological evolution. Moreover, we discuss the conditions under which winding states can play a major cosmological role and solve the stability problem of the compactification radius. Section 4 provides a summary

\section{Thermodynamics of torus-compactified heterotic strings}

It has been demonstrated by various authors (Polchinsk1 1986, Carlip 1986, O'Brien and $\operatorname{Tan} 1987$ ) that the free energy of an ideal string gas is identical to that of an ideal gas of point particles with the corresponding mass spectrum In particular, a modular invariant representation has been found by O'Brien and Tan (1987). This analysis need not be repeated here.

The analogue gas representation is the most convenient for our purpose. We therefore need to know the mass spectrum of the heterotic string compactified on some 6-manifold As an example we shall study torus compactification It does not lead to phenomenologically acceptable models because the compactified theory shows $N=4$ supersymmetry and therefore no chiral fermions. However, no completely satisfactory classical solution is so far known; therefore we may as well study this simple compactification scheme which is well understood. The mass spectrum depends on the background metric $\hat{g}_{i j}$ of the 6-torus and possible other background fields (Narain et al $1987 \mathrm{~b}$ ). For the purpose of demonstration we simply choose $\hat{g}_{l j}=\delta_{i j}$ and put other background fields equal to zero The resultıng mass spectrum is

$$
{ }_{\frac{1}{4}} M^{2}=\frac{1}{4} M_{\mathrm{L}}^{2}+\frac{1}{4} M_{\mathrm{R}}^{2}
$$

with

$$
\begin{aligned}
& \frac{1}{4} M_{\mathrm{L}}^{2}=N_{\mathrm{L}}-1+\frac{1}{2} L^{2} \\
& \frac{1}{4} M_{\mathrm{R}}^{2}=N_{\mathrm{R}}+\frac{1}{2} \tilde{L}^{2}
\end{aligned}
$$

and

$$
\begin{aligned}
& L^{2}=\sum_{i=1}^{6}\left(\left(n^{\prime} R_{l}\right)^{2}+n^{\prime} m_{l}+\frac{1}{4} \frac{m_{l}^{2}}{R_{l}^{2}}\right)+\sum_{I}\left(P^{l}\right)^{2} \\
& \tilde{L}^{2}=\sum_{i=1}^{6}\left(\left(n^{\prime} R_{l}\right)^{2}-n^{\prime} m_{l}+\frac{1}{4} \frac{m_{l}^{2}}{R_{l}^{2}}\right) .
\end{aligned}
$$

Units are such that the string tension $\left(2 \pi \alpha^{\prime}\right)^{-1}$ has the value $1 / \pi$, i.e. $\alpha^{\prime}=\frac{1}{2}$. The $P^{\prime}$ define the $E_{8} \times E_{8}$ root lattice of the left-moving bosonic string sector (Gross et al 
1985). The integers $n^{\prime}$ and $m_{1}$ have the meaning of winding numbers and quantised momenta on the 6-torus respectively, with $R$, the corresponding radii. The oscillator modes $N_{\mathrm{L}}$ and $N_{\mathrm{R}}$ take on non-negative integer eigenvalues. Physical states have to satisfy the constraint

$$
M_{\mathrm{L}}^{2}=M_{\mathrm{R}}^{2}
$$

It is convenient to split the spectrum into non-winding $\left(n^{\prime}=0, \forall 1\right)$ and winding states $\left(n^{k} \neq 0\right.$ for some $\left.k\right)$. Non-winding states have masses

$$
M_{\mathrm{NW}}^{2}=8 N+\sum_{1=1}^{6} \frac{m_{1}^{2}}{R_{1}^{2}}
$$

with $N$ a non-negative integer The contribution of these states to the free energy is

$$
F_{\mathrm{NW}}=-\frac{V_{3} T^{2}}{2 \pi^{2}} \sum_{n=1,3} \frac{1}{n^{2}} \sum_{N=0}^{\infty} d_{N}^{\mathrm{NW}} \sum_{\left\{m_{i}\right\}}\left(8 N+\sum_{i=1}^{6} \frac{m_{l}^{2}}{R_{l}^{2}}\right) K_{2}\left[\frac{n}{T}\left(8 N+\sum \frac{m_{l}^{2}}{R_{l}^{2}}\right)^{1 / 2}\right]
$$

where $d_{N}^{N W}$ is the level degeneracy of the uncompactified ten-dimensional theory In particular there are $d_{0}^{\mathrm{NW}}=8064$ massless states. The asymptotic behaviour is known to be

$$
d_{N}^{N W} \sim N^{-11 / 2} \exp [(2+\sqrt{2}) 2 \pi \sqrt{N}]
$$

determining the critical temperature (1) above which the canonical ensemble does not exist

Equation (6) is the mass spectrum one obtains by first takıng the point field limı in ten spacetime dimensions, and compactify afterwards This procedure misses al] the winding states $\left(n^{k} \neq 0\right.$ for some $k$ ). From (2)-(5) it would be very easy to work out these masses and level degeneracies systematically. This is in fact not even necessary for our purpose, for the following reason. Higher-mass strıng levels give a very small contribution below and even at the critical temperature. For the energy density at a compactification radius $R \sim 1$ (in units $\alpha^{\prime}=\frac{1}{2}$ ) and $T=T_{\mathrm{c}}$ we find a contribution of about $1.2 \%$ It is even smaller for the pressure This is easy to understand because $T_{c}$ (equation (1)) is a small number in units of $\alpha^{\prime}=\frac{1}{2} \quad$ Higher-mass states are therefore exponentially suppressed. In this case it is sufficient for all practical purposes to approximate the non-winding sector of the free energy (7) at $T \leqslant T_{\mathrm{c}}$ by the level $N=0$ states, and concentrate on those winding states which become massless for some values of the compactification scale. It is easy to check that the only states of this type are given by

$$
\begin{array}{ll}
n^{k}=m_{h}= \pm 1 \quad \text { for some } k \\
n^{\prime}=0 & \quad l \neq k \\
\sum_{I}\left(P^{l}\right)^{2}=0 & N_{\mathrm{L}}=N_{\mathrm{R}}=0
\end{array}
$$

i.e. they wind around the $k$ th circle once. The corresponding Kaluza-Klein mass tower is given by

$$
\left(M_{\mathrm{w}}^{(1, k)}\right)^{2}=4 R_{h}^{2}+\frac{1}{R_{k}^{2}}-4+\sum_{i \neq k} \frac{m_{1}^{2}}{R_{i}^{2}}
$$

vanishing for $R_{k}=\bar{R}=1 / \sqrt{2}, m_{l}=0(l \neq k), R_{t}(l \neq k)$ unrestricted. This is a point (or better submanifold) of enhanced symmetry If $R_{l}=\bar{R}$ for all $t$ one gets a maximal 
enhancement

$$
E_{8} \times E_{8} \times U(1)^{6} \Rightarrow E_{8} \times E_{8} \times S U(2)^{6}
$$

The contribution of these winding states to the free energy is

$$
F_{w}=\frac{-V_{3} T^{2}}{2 \pi^{2}} \sum_{n-1,3, \ldots} \frac{1}{n^{2}} 2 d_{0}^{\mathrm{w}} \sum_{k=1}^{6} \sum_{\left\{m_{1}, i \neq k\right\}}\left(M_{\mathrm{w}}^{(1, k)}\right)^{2} K_{2}\left[(n / T) M_{\mathrm{w}}^{(1, k)}\right]
$$

with level degeneracy $d_{10}^{w}=16$, because these states are built on eight degenerate bosonic and fermionic vacuum states. Other winding states are suppressed in (11).

Equation (11) is, however, not appropriate for all purposes. It represents a grand canonical ensemble with zero chemical potential, i.e. assumes unconstrained changes of particle numbers. This is of course not true for the winding states in particular, which can only be pair-produced and cannot decay into non-winding states but only annihilate. Equation (11) should therefore only be used if $R_{k}$ is very close to $\bar{R}$, and these states are copiously produced. Otherwise, if the particle density is low, it is better to use a representation with a fixed number of winding states $N_{k}$. Quantum statistics effects do not play a significant role in this case, and equation (11) should be replaced by

$$
F_{\mathrm{w}}=-T \sum_{k=1}^{5} N_{k} \log \left(\frac{V_{3} T}{2 \pi^{2}} \sum_{\left\{m_{i}, ; \neq k\right\}}\left(M_{\mathrm{w}}^{(1, k)}\right)^{2} K_{2}\left(M_{\mathrm{w}}^{(1, k)} / T\right)\right) .
$$

\section{Cosmological evolution}

We now discuss the influence of winding states on the problem of stability of the compact space. For simplicity we identify the radii $R_{i}=R$ of the 6-torus and study the time evolution of the compactification scale $R$. Denoting the scale factor of 3 -space by $a(t)$ we consider the ten-dimensional metric ansatz

$$
g_{A B}=\operatorname{diag}\left(-1, a^{2}(t), \ldots, R^{2}(t), \ldots\right) \text {. }
$$

The Einstein equations reduce to the following set of differential equations:

$$
\begin{aligned}
& \dot{y}_{1}=\frac{1}{3}\left(-7 \rho+5 p_{3}-6 p_{0}+96 y_{1} y_{2}+120 y_{2}^{2}\right) \\
& \dot{y}_{2}=\frac{1}{3}\left(\rho-3 p_{3}+2 p_{0}-24 y_{1} y_{2}-48 y_{2}^{2}\right) \\
& \dot{\beta}=6\left[\dot{y}_{1}\left(y_{1}+3 y_{2}\right)+\dot{y}_{2}\left(3 y_{1}+5 y_{2}\right)\right]\left(\frac{\partial \rho}{\partial \beta}\right)^{-1}
\end{aligned}
$$

with $\beta(t)=T^{-1}(t)$ the inverse temperature. We have introduced the notation

$$
y_{1} \equiv \dot{a}(t) / a(t) \quad y_{2} \equiv \dot{R}(t) / R(t)
$$

Energy density $\rho$ and 3- and 6-pressure $p_{3}$ and $p_{6}$ are calculated in the standard way from the free energy (equations (7), (11) and (12))

$$
\begin{aligned}
& \rho=\frac{1}{V_{3} V_{6}} \frac{\partial}{\partial \beta}(\beta F) \\
& p_{3}=-\frac{1}{V_{6}} \frac{\partial F}{\partial V_{3}} \\
& p_{6}=-\frac{1}{6} \frac{R}{V_{3} V_{6}} \frac{\partial F}{\partial R}
\end{aligned}
$$


with $V_{6}=(2 \pi R)^{6}$. The constraint equation

$$
3 y_{1}^{2}+18 y_{1} y_{2}+15 y_{2}^{2}=\rho
$$

has to be satisfied initially.

Note that the ten-dimensional gravitational constant $\chi^{2}=8 \pi G_{10}$, which would normally appear in (14) and (17) has been absorbed by a rescaling of time $t \rightarrow \chi^{-1} t$.

The time evolution of the scale factors $a(t), R(t)$ is now completely specified. The choice of initial conditions at $T=T_{\mathrm{c}}$ is less clear, however. Assuming, e.g., a first-order phase transition at $T=T_{c}$ one could imagine the visible part of the universe originating from a single bubble formed during that phase transition. Kinetic considerations would allow us to estimate the bubble size if one could understand the dynamics of the high-temperature phase. Since this is not the case we shall take the initial value of the compactification scale $R$ as a free parameter instead. The stability problem of compactification may be considered as being solved naturally if $R(t)$ does not blow up for a reasonable sufficiently broad range of initial values $R(0)$.

We now discuss the time evolution of $R(t)$. The driving force for a possible blow up of $R$ is $\rho-3 p_{3}+2 p_{6}$ (compare with $(14 b)$ ) which competes with a friction term. We first ignore the winding states. There are two limiting regimes (for $T \leqslant T_{c}$ ):

$$
\begin{array}{ll}
R \ll T^{-1} & \rho \simeq 3 p_{3} \quad p_{6} \approx 0 \\
R \gg T^{-1} & \rho \simeq 9 p_{3} \approx 9 p_{6}
\end{array}
$$

obviously corresponding to a radiation-dominated four-dimensional $(R(t) \rightarrow$ constant, $\left.a(t) \sim t^{1 / 2}\right)$ or ten-dimensional $\left(T(t) \sim a(t) \sim t^{1 / 5}\right)$ expansion, respectively. The range of attraction of these limiting regimes can be found by numerically studying the space of trajectories. In particular, for a symmetric initial expansion rate $\left(y_{1}(0)=y_{2}(0)=\right.$ $\left.1 / 6 p^{1 / 2}\right)$ we find the universe developing towards an effective four-dimensional spacetime if

$$
R(0) \leqslant R^{*} \sim 0.95
$$

At $R=R^{*}, T=T_{\text {c }}$ we find as equations of state

$$
p_{3}=0.27 \rho \quad p_{6}=0.03 \rho \text {. }
$$

Excited string modes have very little influence on this behaviour. The destabilising effect for $R(0)>R^{*}$ arises from the $N=0$ Kaluza-Klein modes (compare with (6)).

Starting from a string theory one also finds higher-derivative contributions to the Einstein equations. Below $T_{\mathrm{c}}$ contributions are also numerically small (Kripfganz and Perlt 1987).

If $R(0)$ is in the stability range $(18), R(t)$ will approach a static value which is stable as long as the universe is radiation dominated. However, when it becomes matter dominated $\left(p_{3} \simeq 1 / 3 \rho \Rightarrow p_{3} \ll \rho\right) y_{2}=0$ is no longer a solution as is obvious from (14b). $R(t)$ will start blowing up. This has been studied, e.g., by Weiss (1986) and need not be repeated here. It would lead, e.g., to a non-acceptable time variation of gauge coupling constants.

In the remaining part of this section we study the question of whether the contribution of winding states to the energy-momentum tensor would qualitatively change this picture. Certainly this can only occur if the number density of winding states at the time $t_{M}$ of the onset of matter dominance is sufficiently large (this will be further specified below). This role cannot be played by strings originally present (they would be totally diluted) but by winding-antiwinding pairs in thermal equilibrium. This 
immediately requires their mass to be small (not much larger than the temperature), which in turn means that $R\left(t_{\mathrm{M}}\right)$ must be extremely close to $\bar{R}$. This would appear unnatural if $\bar{R}$ itself would not act as an attractor. In fact it may do so. The argument has two steps. One is to show that $R(t)$ stays close to $\bar{R}$ up to $t_{\mathrm{M}}$ if it is close to it at a time when $T$ has fallen somewhat below the critical temperature such that the Kaluza-Klein modes are also sufficiently suppressed. In this case the equations of state will be

$$
\begin{aligned}
& p_{3}=\frac{1}{3}+\mathrm{O}\left(r^{2}\right) \\
& p_{6}=-C_{1} T^{2} r+\mathrm{O}\left(r^{3}\right)
\end{aligned}
$$

with $r(t) \equiv R(t)-\bar{R}$. Equation (20) follows from a low-mass expansion of the free energy (7) and (11). The constant $C_{1}$ is given by

$$
C_{1}=3 \pi^{2} d_{0}^{\mathrm{w}} /(2 \pi \bar{R})^{6}
$$

The linearised term of the evolution equation (14b) becomes

$$
8 r+24 y_{1} r+2 \bar{R} C_{1} T^{2} r=0 .
$$

Making use of the constraint equation (17)

$$
\rho \simeq C_{2} T^{4} \simeq 3 y_{1}^{2}
$$

with

$$
\begin{aligned}
& C_{2}=\frac{1}{30} \pi^{2 \frac{15}{16}} d_{0} /(2 \pi \bar{R})^{6} \\
& d_{0}=d_{0}^{\mathrm{NW}}+12 d_{0}^{\mathrm{w}}=8256 .
\end{aligned}
$$

Equation (22) can be solved explicitly by introducing $z=y_{1}^{-1 / 2}$ as a new independent variable Solutions are found to be zeroth-order spherical Bessel functions in $z$, or

$$
R(t) \simeq \bar{R}+\left(r_{1} / \sqrt{t}\right) \sin (2 C t)^{1 / 2}+\left(r_{2} / \sqrt{t}\right) \cos (\sqrt{2 C t})^{1 / 2}
$$

with

$$
C=(\sqrt{3} / 4) \bar{R} C_{1} C_{2}^{-1 / 2}
$$

Therefore, as soon as $R$ is sufficiently close to $\bar{R}$ it will stay close to it during the whole radiation-dominated period Winding string states would be light and abundant.

This conclusion only holds, however, if $R$ gets very close to $\tilde{R}$ in the first place. In general this will not be the case. Numerical calculations show that near the critical temperature the winding contribution (11) to the free energy is so small that $\bar{R}$ does not act as an attractor for a sizeable neighbourhood. A typical trajectory starting with some $R(0)<R^{*}$ will move towards some limiting value different from $\bar{R}$. In this case winding states would be heavy and would not be produced in large numbers. In this case they could not play any role in solving the stability problem of the matterdominated period

There is a way out if the net number of winding strings is non-zero initially. This is not as artificial as it may sound, since we already assume that something like bubbles of a non-trivial topology may form at the critical temperature. This would be quite natural if topology would not be a meaningful concept in the high-temperature phase, e.g. If spacetime becomes discrete. 
Now, if the net number of winding strings would be non-zero initially, and if $R$ is not very close to $\bar{R}$, there would still be a few heavy winding states which cannot decay since they are topologically stable. Equation (12) for the winding sector of the free energy now becomes appropriate. If there are $N_{\mathrm{w}}$ winding states of mass $M_{\mathrm{w}}$

$$
M_{\mathrm{w}}^{2}=4 R^{2}+1 / R^{2}-4
$$

they give a contribution to the pressure $p_{6}$ (for $M_{\mathrm{w}} \geqslant T$ )

$$
p_{6} \simeq-\frac{N_{\mathrm{w}}}{6} \frac{1}{V_{3}(2 \pi R)^{6}} R \frac{\partial M_{\mathrm{w}}}{\partial R}
$$

which drives $R(t)$ towards $\bar{R}$. This contribution will be relevant only for some initial period, until the non-matching winding states are diluted This would be sufficient, however, to give $\bar{R}$ a broad range of attraction. Once $R(t)$ is close to $\bar{R}$ it would follow the solution (25)

There might be other ways of ensuring a density of light winding states of perhaps a few orders of magnitude below the photon number density during the radiation. dominated period. Turning now to matter dominance $R(t)$ will increase somewhat from $\bar{R}$ making these states heavy. Since they are topologically stable they cannot decay but must annihilate. Annihilation will be in general incomplete. Therefore, a small fraction of these states will survive. Effectively, we are again in a situation of a fixed number of winding states and (28) applies. Again we find a stabilising force preventing $R(t)$ from blowing up, provided

$$
\rho+2 p_{6} \leqslant 0 \text {. }
$$

Due to the peculiar $R$ dependence of the mass of the winding states (equation (27)) their energy density may be negligible compared to $2\left|p_{6}\right|$. This will be the case for

$$
R-\bar{R} \leqslant r^{*}=\frac{1}{3 \sqrt{2}} \quad\left(\alpha^{\prime}=\frac{1}{2}\right) .
$$

From equation (28) we finally find a lower bound on the number density $n_{\mathrm{w}}$ of the winding states compared to the baryon density $n_{B}$

$$
\frac{2}{3} n_{\mathrm{w}} \geqslant n_{\mathrm{B}} M_{\mathrm{B}} \sqrt{2 \alpha^{\prime}}
$$

where $\sqrt{2 \alpha^{\prime}} M_{\mathrm{B}}$ is the proton mass in Planck units! The number density of the winding state surviving until the present may therefore be very small, but still provides a stabilisation of the compactification scale.

\section{Summary and díscussion}

The cosmological scenario outlined in this paper for a torus-compactified heterotis string theory has various general aspects valid for non-simply-connected manifolds. At low energies these theories contain additional states due to the compactification They are topologically stable and have a mass depending on the compactification scale $R$. The mass $M_{\mathrm{w}}$ will be minimal for some value $\bar{R}$ of $R$. Interesting cosmological effects are expected in particular if $M_{\mathrm{w}}(\bar{R})=0$. This appears to be a rather general phenomenon at least for torus or orbifold compactificatıons (Narain et al 1987b, Nair et al 1987). Whereas the ground state is scale invariant in these cases the incoherent 
contribution of the winding states to the energy-momentum tensor breaks scale invariance Under reasonable assumptions this contribution stabilises the compactification scale during the cosmological evolution while it would blow up otherwise during the matter-dominated period.

It would be interesting to study the astrophysical consequences of similar but more realistic compactification schemes in some detall As far as we can see, however, these winding states might not have any other observable effect as long as they belong to a h1dden sector, 1.e. the usual matter fields are singlets under the corresponding symmetry enhancement

\section{Acknowledgment}

One of us (JK) would like to thank the DESY theory group for support and kind hospitality.

\section{References}

Alvarez E 1986 Nucl Phys B 269596

Alvarez E and Osorio M A R 1986 Preprint CERN TH 4571/86

Antoniadis I, Bachas C and Kounnas C 1986 Ecole polytechnique preprint A76 11286

Bowick M J and Wijewardnana L C R 1985 Phys Rev Lett 542484

Carlıp S 1986 Texas University preprint Loop calculations for the Green-Schwarz Superstring

Dixon L, Harvey J, Vafa C and Witten E 1985a Nucl Phys B 261678

— 1985b Nucl Phys B 274285

Gle1ser M and Taylor J G 1985 Phys Lett 164B 36

Green M B, Schwarz J H and Witten E 1987 Superstring Theory (Cambridge Cambridge University Press) Gross D J, Harvey J A, Martınes E and Rohm R 1985 Nucl Phys B 256253

- 1986 Nucl Phys B 26775

Hagedorn R 1965 Nuovo Cimento Suppl 3147

Kawa1 H, Llewellen D C and Tye S H H 1987 Nucl Phys B 2881

Kripfganz J and Perlt H 1987 Acta Phys Polon B to be published

Lerche W, Lust D and Schellekens A N 1986 Preprint CERN TH 4590/86

Matsuo N 1986 Osaka University preprint OU-HET-97

Moore G 1987 Harvard University preprint HUTP-87/A013

Nair V P, Shapere A, Strominger A and Wilczek F 1987 Nucl Phys B 287402

Narain K S, Sarmardi M H and Vafa C 1987a Nucl Phys B 288551

Narain K S, Sarmard. M H and Witten E 1987b Nucl. Phys B 279369

O'Brien K H and Tan C I 1987 Brown University preprint BROWN HET.602

Polchınski J 1986 Commun Math Phys 10437

Tye S H H 1985 Phys Lett 158B 388

Weiss N 1986 Phys Lett 172B 180 